\title{
Deutsche zeitgeschichtliche Jugendliteratur der letzten Jahre
}

\author{
Jana Baroková
}

This article deals with the German historical juvenile literature of the last decade, especially with the topic of the Second World War. In the first paragraph, terms like „contemporary historical juvenile literature“, or „the literary communication among different generations“ are discussed. The second part of this article focuses on the writing of the German juvenile novelists Peter Härtling and Gudrun Pausewang. Their partly autobiographic juvenile novels Überleben and Reise gegen den Wind are analyzed. The most important topic in these books is the expulsion of German citizens from Czechoslovakia after the Second World War in 1945.

German contemporary historical juvenile literature - Second World War - expulsion of Germans - Peter Härtling - Gudrun Pausewang - autobiographical novels

Dieser Artikel befasst sich mit der deutschen zeitgeschichtlichen Jugendliteratur des letzten Jahrzehnts, vor allem mit dem Thema des Zweiten Weltkriegs. Zuerst werden Fachtermini wie z. B. „zeitgeschichtliche Kinder- und Jugendliteratur“ oder ,intergenerationelle literarische Kommunikation “erläutert. Das hauptsächliche Augenmerk ist auf das jugendliterarische Werk von Gudrun Pausewang und Peter Härtling gerichtet. Bei diesen Autoren habe ich mich vor allem der Bewältigung des Themas „Vertreibung“ anhand der teilweise autobiographischen Werke Überleben und Reise gegen den Wind gewidmet.

Deutsche zeitgeschichtliche Kinder- und Jugendliteratur - intergenerationelle literarische Kommunikation - Zweiter Weltkrieg - Vertreibung - Peter Härtling - Gudrun Pausewang autobiographische Kinder- und Jugendliteratur

\section{Einführung}

Historische Kinder- und Jugendliteratur als eines der Genres der realistischen Literatur erfreut sich seit einiger Zeit einem wachsenden Interesse bei Lesern und Leserinnen. Laut Gerhard Henke-Bockschatz sind unter dem Begriff historische Kinder- und Jugendliteratur „[...] solche fiktional-literarische Werke zu verstehen, deren Haupthandlung vor dem Hintergrund realer historischer Ereignisse und Verhältnisse geschildert wird“ (HENKE-BOCKSCHATZ: 2008, 197 ff.).

Werke, in denen Themen wie Nationalsozialismus, Zweiter Weltkrieg, Holocaust, Nachkriegszeit und Vertreibung thematisiert werden, sollen der jüngeren Generation diese Begriffe anhand von authentischen Schicksalen aus den Jahren 1933 bis 1945 besser verstehbar machen. Hans-Heino Ewers spricht von einer „intergenerationellen literarischen Kommunikation", weil sich in den autobiographiebasierten zeitgeschichtlichen Romanen für Kinder und Jugendliche eine ältere an eine jüngere Generation wendet. Seitens der Autoren solcher Literatur handelt es sich in der Mehrheit um Angehörige der so genannten zweiten Generation, deren Eltern Täter und Mitläufer des Nazi-Regimes waren. Als Leserschaft angesprochen wird die so 
genannte dritte Generation, also die Enkel der Täter- und Mitläufergeneration (vgl. EWERS/GREMMEL: 2008, 27 ff.).

Mit dem Begriff „Zeitgeschichte“ ist nach Malte Dahrendorf die jüngste oder jüngere Vergangenheit gemeint, in deren unmittelbaren Auswirkung wir heute leben (vgl. DAHRENDORF: 1997, zit. in EWERS/GREMMEL: 2008, 27). In der zeitgeschichtlichen Jugendliteratur tauchen vermehrt Werke auf, die von der Bedeutung der Vergangenheit für die Gegenwart handeln. Neben einer einfachen zeitgeschichtlichen Erzählung, die ausschließlich in der erinnerten Vergangenheit spielt, wie z. B. im Roman Maikäfer flieg! von Christine Nöstlinger, finden wir mehrschichtige zeitgeschichtliche Werke, in denen neben der Ebene der erzählten Vergangenheit eine zweite, in der Gegenwart sich abspielende Handlung dargestellt wird, wie z. B. in der Erzählung Besuch aus der Vergangenheit von Renate Welsh.

In den folgenden Ausführungen werde ich mich überwiegend dem Thema der Vertreibung widmen, die nach dem Ende des Zweiten Weltkrieges stattfand und in der teilweise autobiographischen Erzählweise im Werk von Gudrun Pausewang und Peter Härtling verarbeitet wird.

\subsection{Gudrun Pausewang}

Die im ostböhmischen Wichstadtl (tschechisch Mladkov) 1928 geborene Autorin wuchs als älteste Tochter mit fünf jüngeren Geschwistern in der Familie eines Diplomlandwirts auf, der gemeinsam mit seiner Frau und den Kindern in einem Gutshof auf dem Lande seine Vorstellungen von ökologischer Landwirtschaft und alternativem Leben verwirklichen wollte. 1980 veröffentlicht G. Pausewang im Otto Meier Verlag in Ravensburg das Buch Rosinkawiese als „ein Dokument einer Erfahrung". Sie stilisiert das Buch als Korrespondenz der Tante Elfriede (die Mutter von Gudrun Pausewang) an Michael, den Enkelsohn ihrer Freundin, und vermittelt ihm ihre Erinnerungen an ihre Jugend auf der „alternativen Farm“ als ein langjähriges Experiment, das trotz des letztendlichen Erfolgs sie und ihren Mann viel Kraft und Mühe gekostet hat. Gudrun Pausewang war damals siebzehn Jahre alt, als ihre Mutter mit den Kindern ohne Vater - der in russischer Kriegsgefangenschaft war - die Rosinkawiese verlassen musste. Das Thema der Vertreibung wird in diesem Werk nicht explizit angesprochen. Die Autorin erzählt in einem fiktiven Brief an Michael ihre auf der Rosinkawiese verbrachte Kindheit und dankt ihren Eltern, wie sie sie und ihre Geschwister für das Leben vorbereitet haben:

Meine Eltern haben mir die beste Vorbereitung auf das Leben geboten, die ich mir nur denken kann. Meine Geschwister und ich haben auf der Rosinkawiese gelernt, ohne Komfort auskommen zu können, wir haben gelernt, in Notsituationen nicht den Kopf zu verlieren, sondern nach einem Ausweg zu suchen und durchzuhalten, zu improvisieren, unsere Ansprüche auf ein Mindestmaß herunterzuschrauben. Wir 
haben gelernt, denen, die mehr als wir besaßen, ohne Neid zu begegnen. [Pausewang 2005, 131]

\subsubsection{Jugendbücher zum Thema Nationalsozialismus im Schaffen von Gudrun Pausewang}

Neben den Büchern mit den Hauptthemen wie Frieden, Umweltschutz und Ökologiebewegung, in denen die Autorin klare und deutliche politische Standpunkte verteidigt, schreibt sie seit Anfang der 1990er Jahre eine Reihe von Jugendbüchern zum Thema Nationalsozialismus, wovon einige teilweise autobiographische Züge tragen. Gudrun Pausewang proklamiert selbst, dass diese Zeit nicht vergessen werden darf. So gibt sie sich in der Geschichtensammlung mit dem Titel „Ich war dabei. Geschichten gegen das Vergessen" als Zeitzeugin zu erkennen und bekennt sich gleichzeitig zu den Tätern. In einigen Erzählungen erkennt der Leser, dass die Autorin ihr eigenes Leben schildert. Sie äußert sich ehrlich dazu, dass sie als Jugendliche stolz war, eine Deutsche zu sein. (vgl. EWERS/GREMMEL: 2008, 33 ff.).

Die Handlung des Romans Du darfst nicht schreien (2000) spielt sich während des Sommers 1942 in Brünn ab - nach dem Attentat auf den deutschen Reichsprotektor Reinhard Heydrich. Der Protagonist - Georg, ein deutscher Gymnasiast - findet Unterkunft im Hause einer tschechischen Familie, das sich neben seinem einstigen Internat befindet. Die Vermieter warnen ihn immer wieder davor, die Rollläden auf der Südseite zu öffnen. Als Georg eines Tages jemand in Todesangst schreien hört, und kurz danach Schüsse fallen, bricht er sein Versprechen und schaut durch die Rollläden:

Plötzlich dämmerte es Georg, was dort drüben stattfand. Er riss die Augen auf und öfnete den Mund. Aber noch bevor er einen Schrei ausstoßen konnte, krachte die nächste Salve. Er zog den Kopf zwischen die Schultern und schloss die Augen. Als er sie wieder zu öfnen wagte, war die Frau verschwunden.

Georg war wie gelähmt. Er ließ das Rollo los, das mit einem Seufzer zurückschnellte.

Benommen schloss er das Fenster, schwindlig von irren Gedanken. Ihm war, als müsste ihm der Kopf bersten. Auf halbem Weg zu seinem Bett übergab er sich auf das Parkett. Ihn beherrschte nur ein einziges Gefühl: Entsetzen. Taumelnd erreichte er das Bett, rollte sich darauf zusammen wie ein Embryo, schloss die Augen und hielt sich in Erwartung weiterer Schüsse die Ohren zu.

Noch eine Salve. Und noch eine. Und noch eine. Dazwischen quälende Pausen und einzelne Schüsse. Dann Stille.

Oh, ich Idiot, dachte er, Schießübungen! [Pausewang 2000, 80] 
In der Korrespondenz mit einer Studentin der Pädagogischen Fakultät in Brünn äußert sich Gudrun Pausewang zu diesem Buch ausführlich. Nach Meinung der Autorin war ihr Buch in Deutschland nur mäßig erfolgreich. Das lag vor allem am Thema. In den Jahren, als es erschien, war in der deutschen Öffentlichkeit nur wenig Interesse an Geschichten aus der Nazizeit vorhanden. Die Autorin äußerte sich weiter dazu, wie sie zum Thema des Werkes kam. Den Inhalt des Romans Du darfst nicht schreien hat der Autorin auf einer Lesung ein pensionierter Ingenieur erzählt, der einst Schüler eines Brünner Gymnasiums war und während der Schulzeit im Schulinternat wohnte. Als dieses Internat geschlossen wurde, weil es zu einem Lazarett umfunktioniert wurde, fand er ein Zimmer in der Nähe des Kaunitz-Kollegs. Dort hörte er an jedem frühen Nachmittag, während er seine Hausaufgaben machte, die Schüsse und kam dahinter, was sie bedeuteten: Dort wurden Nazigegner und Widerstandskämpfer von der Gestapo hingerichtet. ${ }^{1}$ Als Gudrun Pausewang Stoff für diesen Roman sammelte, besuchte sie in Brünn das Widerstandsmuseum im Kaunitz-Kolleg und erhielt vom Museumsverwalter Fotokopien von Nazidokumenten. Später besorgte sie sich in Prag historische Schilderungen der Geschichte des „Protektorats“ zwischen März 1939 und Mai 1945, wobei sie sich vor allem für die Zeit nach dem Attentat auf den Reichsprotektor Reinhard Heydrich interessierte.

\subsubsection{Thema der Vertreibung im Roman Überleben von Gudrun Pausewang}

In diesem im Jahre 2005 veröffentlichten Roman wendet sich die Autorin in der Rolle einer Großmutter an ihre Enkelin Stefanie, der sie anlässlich ihres sechzehnten Geburtstags ihre eigene Geschichte schenken möchte - wie es war, als sie 1945 selbst sechzehn Jahre alt wurde und mit ihrer Mutter, Großmutter und jüngeren Geschwistern aus dem Land ihrer Jugend nach Deutschland floh. Es fängt ein spannender Bericht über Flucht- und Bombenkriegserlebnisse an:

Verdammter Krieg! "Verdammt“ darf man zwar nicht sagen. Aber ich finde kein anderes Wort für ihn. Wenn Frieden wäre, hätten wir nicht alles daheim lassen müssen. Wenn Frieden wäre, hätten wir gar nicht Hals über Kopf unser Haus, unser Dorf verlassen müssen! Wenn kein Krieg wäre, hätten die Russen nicht den größten Teil von Schlesien besetzt. Dann wäre Schlesien ein schönes Stück Deutschland, friedlich und freundlich. [Pausewang 2005, 10]

Diese Geschichte der Vertreibung basiert teilweise auf der eigenen Autobiographie der Schriftstellerin. Es ist Februar 1945 und aus einem niederschlesischen Dorf flüchtet die sechzehnjährige Gisela mit ihrer Mutter, Großmutter und zwei kleineren

1 Heute befindet sich im ehemaligen Kaunitz-Kolleg (tschechisch „Kounicovy koleje) das Denkmal für den antifaschistischen Widerstandskampf. 
Brüdern - dem sechsjährigen Harald und eineinhalbjährigen Wolfi - vor der immer näher rückenden Ostfront. Die vorher geplante Reise, nach der die fliehende Familie Quartier bei Verwandten im hessischen Ort Bebra finden soll, wird allmählich zu einer dramatischen Abenteuergeschichte, in der es um die Rettung des nackten Lebens geht. Unterwegs wird die hochschwangere Mutter der Protagonistin mit Wehen ins Krankenhaus gebracht und verschwindet vorübergehend aus dem Leben der Kinder. Bei einem Fliegeralarm werden die Kinder, die inzwischen im Menschengewühl auf dem Bahnhof auch ihre Großmutter verloren haben, in einem im Klo eines Bunkers verschüttet. Es folgen unendlich lange Stunden in der Dunkelheit der Toilette des Luftschutzkellers, ausgefüllt mit Leid, Angst ums Leben, Hunger und Durst, bevor die Kinder von den Menschen des Roten Kreuzes gerettet werden und bevor sie schließlich auch ihre Großmutter glücklich umarmen können.

Gudrun Pausewang zeigt in diesem Buch die Deutschen nicht als Opfer des Luftkriegs, sie lässt keinen Zweifel daran, wie und warum es zu diesem Krieg gekommen ist. So führt die Ich-Erzählerin Gisela z. B. innerliche retrospektive Gespräche mit ihren Eltern, um die Frage zu beantworten, wer für diesen Krieg und das Elend verantwortlich ist:

Nein, der Krieg blieb keine Siegesfeier am laufenden Band. „Er ist dem Führer aus dem Griff geraten", hab ich Vati bei seinem letzten Fronturlaub leise zu Mutti sagen hören. „Er hat zu viel erreichen wollen - und das zu schnell. [Pausewang 2005, 17]

Viel mehr als mit dem Thema der Schuld der Deutschen am Zweiten Weltkrieg befasst sich Gudrun Pausewang in diesem Roman mit der Darstellung positiver Charaktereigenschaften der Protagonisten. So z. B. muss Gisela die für die Reise nach Deutschland vorbereiteten Lebensmittel- und Wasservorräte in der zugespitzten Situation nicht nur für sich selbst und ihre jüngeren Brüder gerecht teilen, sondern sie kümmert sich auch um die etwa fünfährige Lotte, ein Mädchen, dessen Mutter im Gedränge auf dem Bahnhof verloren gegangen ist. Mit dem knapp gewordenen Wasser hilft Gisela sogar einem im Nebenraum des Luftschutzkellers verschütteten und langsam sterbenden deutschen Soldaten, sobald sie durch ein Rohr einen geeigneten Weg für seine Wasserversorgung gefunden hat.

Im letzten Kapitel des Buches fasst Gudrun Pausewang in einem Brief an die Enkeltochter Stefanie die ersten Tage und Monate zusammen, die auf die Verschüttung und die Rettung der Kinder folgen und in denen sich die Schicksale der Protagonisten für die erste Nachkriegszeit abrunden. Der Findling Lotte bleibt bei der Familie, weil seit der Verschüttung ihr Schicksal mit demjenigen Giselas und ihrer Geschwister eng verbunden ist: „, [...] zwei Nächte und fast zwei Tage tief im Keller verschüttet zu sein und im dunkeln zu sitzen. Dieses Erlebnis hat sie mit euch geteilt. Das verbindet [...]“, so entscheidet schließlich die Großmutter [Pausewang 2005, 214]. 
Im Juli kommt endlich auch die Mutter, abgemagert, grauhaarig und ohne das neugeborene Kind - es starb, weil sie nicht genug Milch hatte. Der aus der Gefangenschaft nierenkrank zurückgekehrte Vater war anders als vorher:

Unser Nachkriegsvati war jedenfalls ein ganz anderer als unser Vorkriegsvati. Besonders erregbar war er, wenn es in Gesprächen um Schlesien, seine Heimat, ging. Bei jeder Gelegenheit schimpfte er auf die Polen, die - nach seiner Meinung Schlesien ruinierten. [Pausewang 2005, 218]

Wie in anderen ihren Erzählungen über den Zweiten Weltkrieg präsentiert sich die Autorin auch in diesem Buch als Zeitzeugin. Sie stellt den Wahrheitsgehalt ihrer Schilderungen heraus und bekennt gleichzeitig, auch zu den Tätern gezählt zu haben. Für das höchste Ziel hält sie das Wecken der Aufmerksamkeit für jene Kapitel der Geschichte, die aus dem deutschen Bewusstsein verdrängt und eliminiert werden. Sie plädiert dafür, dass die Leser solche Schriftsteller, die die Kriegszeit noch selbst erlebt haben, stetig befragen, solange dazu noch die Möglichkeit besteht (vgl. EWERS/GREMMEL: 2008, 33 ff.).

\subsection{Peter Härtling als Kinder- und Jugendbuchautor}

Peter Härtling wurde 1933 in Chemnitz geboren. Sein Vater - der Rechtsanwalt war - übersiedelte 1941 mit seiner Familie nach Olmütz, um vor den Nazis zu fliehen. Während des Krieges wurde er trotzdem eingezogen und starb 1945 in einem Gefangenenlager in Niederösterreich. Ein Jahr später beging die Mutter Selbstmord.

Peter Härtling fing seine Karriere als Journalist an, seit 1974 ist er freier Schriftsteller. In die Literatur trat er als Lyriker. Seine ersten Gedichtbände sind von der abwehrenden Haltung gegenüber der Nachkriegsentwicklung geprägt, spätere Poesie ist mit Gedanken des Außenseitertums, der Nichtanpassung und mit subjektiven Ängsten durchdrungen. In seiner Prosa, die geschichtliche Ereignisse des 20. Jahrhunderts reflektiert, erinnert er sich an das Vergangene, an Selbsterlebtes, vor allem an die Nazi-Zeit und die Nachkriegsjahre. Im Roman Nachgetragene Liebe (1980) überwiegt das Autobiographische aus den in Olmütz zwischen 1941-1945 verbrachten Jugendjahren, in denen er von der Nazipropaganda und -bewegung der Jugend stark angezogen wurde. Der Autor versucht einen Kontakt zu seinem Vater retrospektiv anzuknüpfen und wirft dem Vater vor, sich damals nicht klarer und eindringlicher ausgedrückt zu haben.

Dem Schreiben der Kinder- und Jugendbücher widmet sich Peter Härtling seit den siebziger Jahren. Die Mehrheit seiner prosaischen Jugendwerke bilden die sog. Problembücher, in denen er die kindlichen Leser auf die Verantwortung und Bewältigung von Schwierigkeiten vorbereitet. Seine Protagonisten miterleben 
gemeinsam mit ihren Eltern Krankheit, Tod, Behinderung, aber auch Arbeitslosigkeit und Scheidung.

Das Kredo seines literarischen Schaffens hat P. Härtling in seiner Rede anlässlich der Verleihung des Deutschen Jugendliteraturpreises im Jahr 1969 prägnant formuliert: „Eine autoritäre Erziehung hat lange Jahre eine autoritäre Literatur geschaffen. Hoch klang das Lied vom braven Kind. Es gab nicht nur den Professor Unrat; es gab auch den Schüler Unrat. Generationen von Eltern halfen mit, seelische und geistige Krüppel ins Leben zu schicken, gelehrige Untertanen, die gelernt hatten, nicht aufzumucken: dem Vater gegenüber nicht, dem Lehrer gegenüber nicht, dem Staat gegenüber nicht. [...] Es ist schwer, dem Kind beizubringen, daßsich Wirklichkeit und Freiheit unaufhörlich verbünden. " (Vgl. Härtling 1969, zit. in SCHIKORSKY: 2003, 156)

\subsection{Das Thema Vertreibung im Jugendroman Reise gegen den Wind von Peter Härtling}

Teilweise eigene Lebenserfahrungen vom Ende des Zweiten Weltkriegs verarbeitet P. Härtling bereits in der 1987 erschienen und später verfilmten Erzählung Krücke. Der 13-jährige Tom verliert in den letzten Kriegstagen seine Mutter und in den Wirren der Flucht verschlägt es ihn nach Wien. Hier begegnet er dem einbeinigen Schwarzhändler „Krücke“ und beide freunden sich an.

In dem im Jahr 2000 erschienenen Roman Reise gegen den Wind wird Härtlings eigenes Kindheitserlebnis - die Vertreibung im Frühjahr 1945 aus Brünn nach Österreich - teilweise autobiographisch dargestellt. Der zwölfährige elternlose Bernd - von seiner Tante Karla zu seinem großen Leidwesen Primel genannt - erlebt die Odyssee der Flucht, als alles verloren ging und die Tage mit Unsicherheit erfüllt waren, als ein großes Abenteuer. In der nicht willkommenen Pause der Suche nach einer neuen Heimat in dem verschlafenen niederösterreichischen Ort Laa an der Thaya - wo deutsche Flüchtlinge einige Tage vergeblich auf einen Zug warten und immer wieder bei den Einheimischen um Quartier bitten müssen - lebt Bernd das Leben eines 12-jährigen Kindes: Er schließt Freundschaft mit zwei Kindern und ein herrenloser Hund findet vorübergehend Obdach unter Bernds Bett. Spannung in die Handlung bringt der geheimnisvolle, „fabelhafte" Herr Maier. Niemand weiß genau, wer dieser immer elegant gekleidete Herr ist. Mit seinem charmanten und höflichen Auftreten knüpft er mühelos Kontakte und hat daher überall Freunde. Es wird gemunkelt, dass er Schwarzhändler sei und krumme Geschäfte mache. Kurz vor der Abfahrt des Zuges nach Wien findet Bernd Herrn Meier erschossen im Wald:

Neben der Hütte mit eingestürztem Dach entdeckte er im Farn einen schwarzen Buckel, etwas Fremdes. Er dachte an die Gräber der Wlassow-Soldaten, an den Fuß, 
der aus der Erde geragt hatte. Er kniff die Augen zusammen, sah zu dem schwarzen Haufen hinüber, trat ein paar Schritte näher, um mehr zu sehen. Schillernde Fliegen hoben sich wie ein Schild von dem Gegenstand, und es roch entsetzlich, süß und klebrig. Der Gestank würgte ihn. Er lag auf dem Bauch, riesig, ein gestürztes Ungetüm, in seinem schwarzen Anzug. Es konnte nur der fabelhafte Herr Maier sein. Auch wenn sein Gesicht sich in den Waldboden drückte. [Härtling 2000, 132]

Die Geschichte ist unsentimental genau, aus der Perspektive des Kindes als ein Stück Zeitgeschichte geschildert. Im letzten Kapitel - genannt Und weiter? - schildert Peter Härtling in sparsamer Ausdrucksweise und kurzen einfachen Aussagesätzen, wie das Leben des Protagonisten Bernd und seiner Tante Karla weiterging, wie in Wien allmählich ihre Einstufung als Flüchtlinge über Neubürger bis schließlich zu Mitbürgern in der Hierarchie der modernen Gesellschaft stieg.

Während der Autor in seinen an erwachsene Leser adressierten Büchern - wie Nachtgetragene Liebe (1980) oder Leben lernen (2003) - gesteht, wie stark er als Kind unter den Einfluss der nationalsozialistischen Ideologie geraten war, bleiben diese Aspekte in seinem kinderliterarischen Werk völlig ausgespart. Diese idealisierende Darstellung der kindlichen Figuren, gemeinsam mit der stereotypen Darstellung der Nationalsozialisten kann jedoch dazu führen, dass heutige Jugendliche die NS-Propaganda unterschätzen (vgl. EWERS/GREMMEL: 2008, 32-33).

\section{Schlusswort}

Das Thema dieses Artikels ist die deutsche zeitgeschichtliche Jugendliteratur der letzten Jahre. Die größte Inspiration zu seiner Abfassung war für mich der im Frühjahr 2005 an der Universität in Frankfurt am Main stattgefundener Weltkongress zum Thema „Die Generation der Kriegskinder und ihre Botschaft für Europa sechzig Jahre nach Kriegsende“. Der im Jahre 2008 erschienene Sammelband, der Beiträge von Jugendliteraturforscherinnen und -forschern aus der ganzen Welt enthält, fasst sowohl theoretische Ausführungen als auch Beispiele aus der Primärliteratur zum gegebenen Thema zusammen. Ich habe mich anhand dieser Quelle zuerst mit Begriffen wie „Zeitgeschichte“, „historische Kinder- und Jugendliteratur“ oder „intergenarationelle literarische Kommunikaton“ befasst. Auf dieser Konferenz habe ich auch Frau Gudrun Pausewang kennen gelernt, die mich und meine Studentinnen und -studenten zur Lektüre ihres Werkes anregte. Ihr teilweise autobiographisches Werk über ihre Erlebnisse aus dem Zweiten Weltkrieg, vor allem das Thema der Vertreibung, bildet den zweiten Teil dieses Artikels. Ein ähnliches Schicksal wie das der Autorin hatte nach dem Ende des Zweiten Weltkrieges auch Peter Härtling. Mit der Analyse der Werke Überleben von Gudrun Pausewang und Reise gegen den Wind von Peter Härtling habe ich zu zeigen versucht, wie beide Autoren diesen Stoff in ihren kinderliterarischen Werken bewältigt haben. 


\section{Primärliteratur:}

HÄRTLING (2000): Härtling, Peter. Reise gegen den Wind. Weinheim Basel: Beltz \& Gelberg, 2000.

PAUSEWANG (1980): Pausewang, Gudrun. Rosinkawiese. Alternatives Leben vor 50 Jahren. Ravensburg: Otto Meier Verlag Ravensburg, 1980.

PAUSEWANG (2005): Pausewang, Gudrun. Überleben. Ravensburg: Ravensburger Buchverlag Otto Meier GmbH, 2005.

PAUSEWANG (2000): Pausewang, Gudrun. Du darfst nicht schreien. Ravensburg: Ravensburger Buchverlag Otto Meier GmbH, 2000.

\section{Sekundärliteratur:}

EWERS/GREMMEL (2008): Ewers, Hans-Heino/Gremmel, Caroline. Zeitgeschichte, Familiengeschichte und Generationenwechsel. Deutschezeitgeschichtliche Jugendliteratur der 1990er und 2000er Jahre im erinnerungskulturellen Kontext, in: Glasenapp von, Gabriele/Ewers, Hans-Heino (Hg.): Kriegs- und Nachkriegskindheiten. Studien zur literarischen Erinnerungskultur für junge Leser. Frankfurt am Main: Peter Lang, 2008, $27-50$.

HENKE-BOCKSCHATZ (2008): Henke-Bockschatz, Gerhard. Zeitgeschichtliche Kinderund Jugendliteratur als Medium historischen Lernens, in: Glasenapp von, Gabriele/ Ewers, Hans-Heino (Hg.): Kriegs- und Nachkriegskindheiten. Studien zur literarischen Erinnerungskultur für junge Leser. Frankfurt am Main: Peter Lang, 2008, 197-215.

SCHIKORSKY (2003): Schikorsky, Isa. Kinder-und Jugendliteratur. Köln: DuMont Literatur und Kunst Verlag, 2003. 